\title{
Gallic Acid Potentiates the Antimicrobial Activity of Tulathromycin Against Two Key Bovine Respiratory Disease (BRD) Causing-Pathogens
}

\section{OPEN ACCESS}

Edited by

Banasri Hazra,

Jadavpur University, India

Reviewed by:

Diganta Dey,

Ashoke Laboratory Clinical Testing Centre Private Limited, India

Timothy John Mahony, The University of Queensland,

Australia

*Correspondence:

Jian Yang

jian.yang@usask.ca

Meena Kishore Sakharkar meena.sakharkar@usask.ca

Specialty section: This article was submitted to

Ethnopharmacology,

a section of the journa

Frontiers in Pharmacology

Received: 20 July 2018 Accepted: 04 December 2018 Published: 04 January 2019

Citation:

Rajamanickam K, Yang J and Sakharkar MK (2019) Gallic Acid Potentiates the Antimicrobial Activity of Tulathromycin Against Two Key Bovine Respiratory Disease (BRD)

Causing-Pathogens.

Front. Pharmacol. 9:1486. doi: 10.3389/fphar.2018.01486
Karthic Rajamanickam, Jian Yang* and Meena Kishore Sakharkar*

Drug Discovery and Development Research Group, College of Pharmacy and Nutrition, University of Saskatchewan, Saskatoon, SK, Canada

Bovine respiratory disease (BRD) is the most common infectious disease in dairy and beef cattle. It is associated with significant morbidity and mortality and causes a huge economic loss each year. In western Canada, a one-time injection of tulathromycin is commonly used as a metaphylactic procedure to reduce BRD incidence and eliminate potential BRD outbreak. With increased global concern on antimicrobial usage in dairy and beef products and bacterial resistance to antimicrobials, it is important to develop a novel strategy to eliminate the usage or decrease the dosage of antimicrobials. In this study, we showed that gallic acid was active against both Mannheimia haemolytica and Pasteurella multocida, two key BRD associated-pathogens, with the minimum inhibitory concentration (MIC) measured at 250 and $500 \mu \mathrm{g} / \mathrm{mL}$, respectively. Coadministration of tulathromycin and gallic acid exhibited a strong additive or weak synergistic effect toward both $M$. haemolytic and P. multocida. Tulathromycin, gallic acid and their combination were also effective against the mixed culture of $M$. haemolytic and P. multocida. Furthermore, we showed that pre-exposure to tulathromycin generated bacterial resistance to the antimicrobial in $M$. haemolytica but not in P. multocida.

Keywords: bovine respiratory disease, gallic acid, tulathromycin, dairy and beef cattle, antimicrobial resistance

\section{INTRODUCTION}

Bovine respiratory disease (BRD) is the most prevalent infectious disease in diary and beef cattle (Griffin, 1997). BRD imposes deleterious effects on cattle health and performance, resulting in substantial economic loss (Wittum et al., 1996; Smith, 1998; Larson, 2005; Rajala-Schultz et al., 2009; Johnson and Pendell, 2017). For example, the annual loss caused by BRD is about \$US 600-750 million to the North American beef industry. BRD is commonly regarded as a multifactorial disease, with bacterial infection, viral infection, and stress as the three major co-dependent factors (Holman et al., 2017; Headley et al., 2018). The key pathogenic bacteria identified in Canada are Mannheimia haemolytica, Pasteurella multocida and Haemophilus somni (Ellis, 2001; Apley, 2006). Vaccines have been developed against these three pathogens; however, the vaccination result is not consistent (Larson and Step, 2012). In western Canada, new feedlot placements usually get a metaphylactic injection of tulathromycin (Draxxin) to reduce the risk and severity of BRD upon arrival (Lindsay, 2003; Checkley et al., 2010). Tulathromycin is a semi-synthetic triamilide antimicrobial, which inhibits the synthesis of essential bacterial proteins (Schunicht et al., 2007). 
The practice of metaphylactic injection of antimicrobials may lead to the development of antimicrobial resistance (AMR), which, in turn, will reduce the efficacy of the antimicrobials commonly employed to control infectious disease, such as BRD, in cattle (Checkley et al., 2010). Here, it is important to mention that subinhibitory concentrations of antimicrobials have been suggested to be associated with generation of genotypic and phenotypic variability and has been used for selection of bacteria resistant to antimicrobials (Andersson and Hughes, 2014). Antimicrobials at subinhibitory concentrations have been reported to function as signaling molecules causing alterations in bacterial physiology e.g., alterations in breakpoint, virulence, biofilm formation, gene transfer, etc (Bhattacharya et al., 2017). Furthermore, with increased concern from the consumers about antimicrobial usage in dairy and beef products, Health Canada has decided to introduce a new regulation that a veterinary prescription is required to purchase any livestock antimicrobial from December (West-Central Forage Association, 2018). Thus, there is an urgent need to identify alternatives of antimicrobials, such as natural products, and develop novel and effective treatment protocols with significantly reduced usage, and dosage of antimicrobials in order to minimize the development of AMR. For example, controlling pulmonary inflammation with a nonsteroidal anti-inflammatory drug (NSAID) is critical to avoid irreversible lung damage in the cattle other than the antimicrobial treatment of BRD (Lindsay, 2003).

Plants synthesize a diverse array of secondary metabolites (phytochemicals), which are not only involved in self-defense but also possess a wide range of health-promoting effects such as antimicrobial activities. The use of antimicrobials in combination with phytochemicals has been extensively studied (Monden et al., 2002; Petersen et al., 2006; Černohorská and Votava, 2008). The antimicrobial-phytochemical cocktail strategy has exhibited the potential in eradicating complex pathogens (Neu, 1991; Batista et al., 1994; Cowan, 1999; Lewis and Ausubel, 2006; Wise, 2006; Amyes et al., 2007; Cai et al., 2007; Gould, 2008). Furthermore, majority of phytochemicals are relatively safe for use as compared to purely synthetic drugs due to their natural origins and can be metabolized easily without leaving harmful residues in dairy and beef products. Co-administration of phytochemicals has been shown to enhance antimicrobial activities of antimicrobials and delay the development of AMR. In this study, we evaluated whether gallic acid, a phenolic acid identified in various plants such as gallnuts, possesses antimicrobial activity, potentiates the antimicrobial function of tulathromycin, and reduces or delays AMR to tulathromycin against two key causing-pathogens of BRD, M. haemolytica and P. multocida.

\section{MATERIALS AND METHODS}

\section{Materials}

Bacterial strains M. haemolytica ATCC 29702 and P. multocida ATCC 43137, as well their culture medium (Brain-Heart Infusion broth, BHIB), were purchased from Cedarlane Canada (Burlington, ON, Canada). Gallic acid was purchased from ThermoFisher Scientific (Ottawa, ON, Canada). Tulathromycin
A was purchased from Cayman Chemical Company (Ann Arbor, MI, United States). All other chemicals used in this study were purchased from Sigma-Aldrich Canada (Oakville, ON, Canada).

\section{Determination of Minimum Inhibitory Concentration (MIC)}

All experiments in this study were carried out in triplicate. The MICs of tulathromycin and gallic acid against $M$. haemolytica and $P$. multocida were determined using standard broth microdilution assay as outlined by the Clinical \& Laboratory Standards Institute (CLSI). Both strains were sub-cultured in BHIB at $37^{\circ} \mathrm{C}$ overnight and then $\mathrm{OD}_{565}$ of the bacterial suspensions was adjusted to $0.5 \mathrm{McF}$ arland turbidity with the culture media (approximate cell density: $1.5 \times 10^{8} \mathrm{CFU} / \mathrm{mL}$ ) using normal saline as a control. For each bacterial strain, $100 \mu \mathrm{L}$ BHIB broth was added to each well of a 96-well plate with subsequent addition of $5 \mu \mathrm{L} /$ well of the adjusted bacterial suspension. Then, the bacterial samples were treated with either tulathromycin with concentration ranging from 0.04 to $5 \mu \mathrm{g} / \mathrm{mL}$ or gallic acid with concentration ranging from 3.9 to $500 \mu \mathrm{g} / \mathrm{mL}$. Untreated bacterial samples were used as a negative control. The culture plate was incubated at $37^{\circ} \mathrm{C}$ for $18-24 \mathrm{~h}$ before $\mathrm{OD}_{655}$ was taken for each well using a Bio-Rad iMark Microplate Reader (Bio-Rad Laboratories, Inc., Mississauga, ON, Canada). The readings were also double-checked using a Sensititre Vizion Digital MIC Viewing System (ThermoFisher Scientific, Ottawa, ON, Canada).

\section{Antimicrobial Effect of the Combination of Tulathromycin and Gallic Acid}

For each bacterial strain, the antimicrobial activity of the combination of tulathromycin and gallic acid was measured a protocol modified from the one described above. Briefly, the bacterial sample preparation in the 96-well plate was the same. Then, tulathromycin and gallic acid were added to the bacterial sample following a checkerboard serial dilution design. The tulathromycin concentration was 0.08 and $0.16 \mu \mathrm{g} / \mathrm{mL}$, respectively; and the gallic acid concentration was from 3.91 to $250 \mu \mathrm{g} / \mathrm{mL}$. The fractional inhibitory concentration (FIC) index of the combination of tulathromycin and gallic acid was calculated according to the equation 1 . In this equation, $\mathrm{FIC}_{\mathrm{A}}$ and $\mathrm{FIC}_{\mathrm{B}}$ are the FIC indices of compounds $\mathrm{A}$ and $\mathrm{B}$ in the combination, $\mathrm{A}$ and $\mathrm{B}$ are the MICs of compounds $\mathrm{A}$ and $\mathrm{B}$ in combination, and $\mathrm{MIC}_{\mathrm{A}}$ and $\mathrm{MIC}_{\mathrm{B}}$ are the individual MICs of compounds $\mathrm{A}$ and $\mathrm{B}$, respectively. The FIC index determined by checkerboard method is interpreted as following: FIC $\leq 0.5$ - synergy; $0.5<$ FIC $\leq 4.0$ - additivity; and FIC > 4.0 - antagonism

$$
\text { FIC index }=\text { FIC }_{\mathrm{A}}+\mathrm{FIC}_{\mathrm{B}}=A / M I C_{\mathrm{A}}+\mathrm{B} / \mathrm{MIC}_{\mathrm{B}}
$$

\section{Evaluation of Bacterial Resistance Generated From Pre-exposure}

In order to evaluate whether bacterial resistance can be generated from pre-exposure to tulathromycin, M. haemolytica, 
and P. multocida were cultured in BHIB in the presence of tulathromycin at a dose of 1/2MIC (i.e., $0.16 \mu \mathrm{g} / \mathrm{mL}$ ) until $\mathrm{OD}_{565}$ reached $1 \mathrm{OD}$ unit of turbidity. Then, the bacterial cells were given a stress relaxation by culturing in BHIB without tulathromycin until $\mathrm{OD}_{565}$ reached $1 \mathrm{OD}$ unit of turbidity. The bacterial cells were collected and designated as the 1st generation of pre-exposed cells $(1 \mathrm{G})$. The $1 \mathrm{G}$ bacterial cells were subjected to the same type of treatment to generate the 2nd generation of pre-exposed cells $(2 \mathrm{G})$; and subsequently the $2 \mathrm{G}$ bacterial cells were subjected to the same protocol to generate the $3 \mathrm{rd}$ generation of pre-exposed cells (3G). In total, we generated three generations (1G, 2G, and 3G) of M. haemolytica and P. multocida cells pre-exposed to tulathromycin. The MICs of tulathromycin and gallic acid against the $1 \mathrm{G}, 2 \mathrm{G}$, and $3 \mathrm{G}$ M. haemolytica and
P. multocida cells were also measured using the protocol describe above.

\section{Determination of MIC Against Mixed Culture of $M$. haemolytica and $P$. multocida}

The MICs of tulathromycin and gallic acid against the mixed culture of $M$. haemolytica and P. multocida were determined using the same protocol described above except that both strains were mixed cultured in $\mathrm{BHIB}$ at $37^{\circ} \mathrm{C}$ overnight and $\mathrm{OD}_{565}$ of the mixed cultured bacterial suspension was adjusted to 0.5 McFarland turbidity with the culture media. The antimicrobial effect of the combination of tulathromycin and gallic acid against

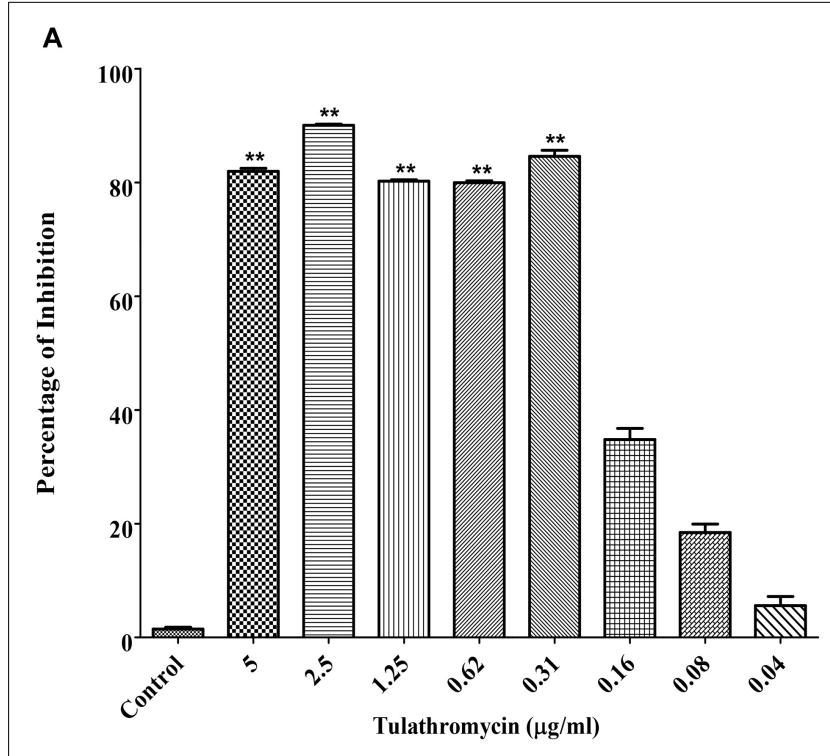

B

C

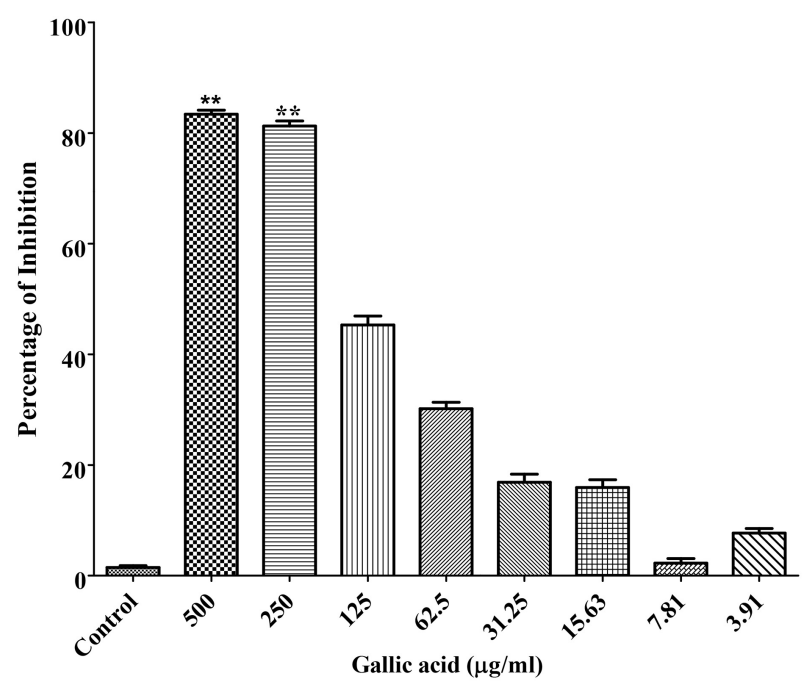

D
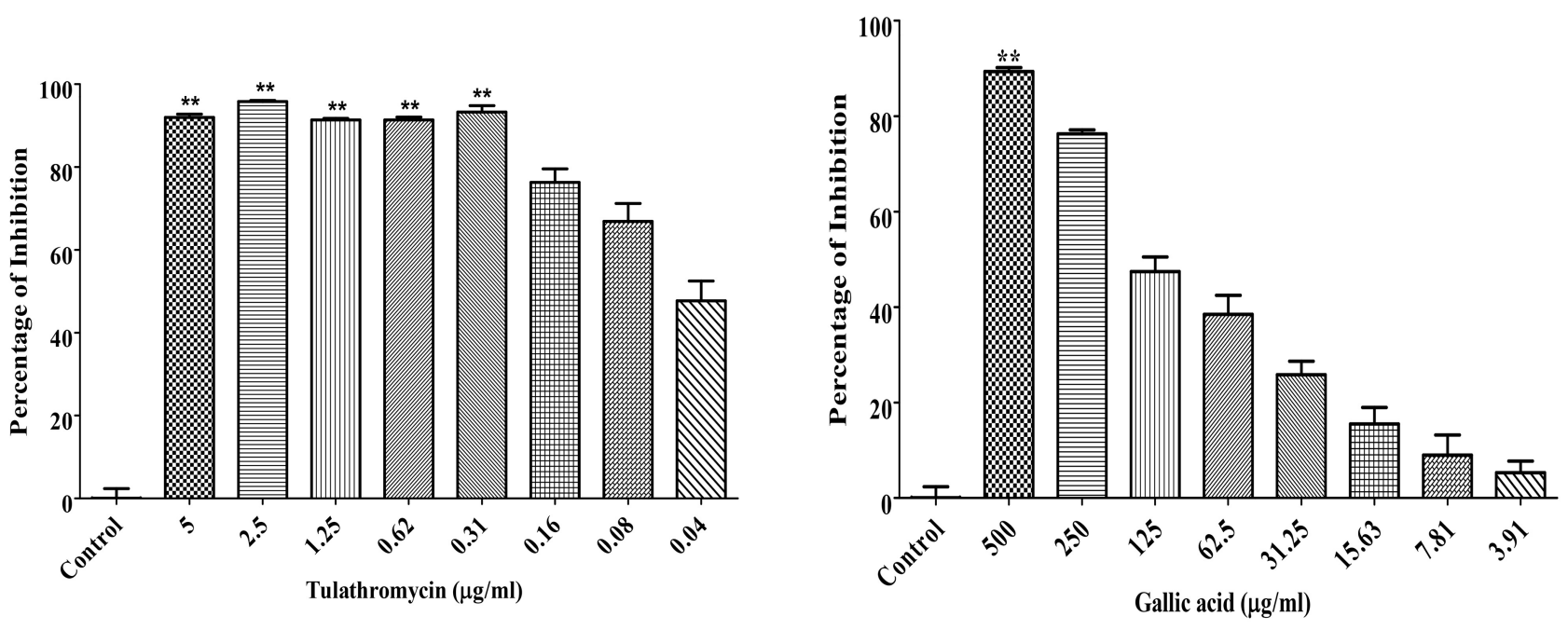

FIGURE 1 | Determination of the MICs of tulathromycin against M. haemolytica (A) and P. multocida (B) and the MICs of gallic acid against M. haemolytica (C) and P. multocida (D). The concentration of tulathromycin was ranging from 0.04 to $5 \mu \mathrm{g} / \mathrm{mL}$ and the concentration of gallic acid was ranging from 3.9 to $500 \mu \mathrm{g} / \mathrm{mL}$. Bacterial cell culture medium, Brain-Heart Infusion broth (BHIB), was used as a negative control. One way ANOVA $t$-test was used for statistical significance. ${ }^{*} P$-value $<0.05,{ }^{* *} P$-value $<0.01$, and ${ }^{* * *} P$-value $<0.001$ are shown. Correlation coefficient $(r)$ was calculated using Pearson's correlation method. 
the mixed culture of M. haemolytica and P. multocida was also measured using the checkerboard serial dilution method. The tulathromycin concentration was $0.16,0.31$, and $0.62 \mu \mathrm{g} / \mathrm{mL}$, respectively; and the gallic acid concentration was from 3.91 to $500 \mu \mathrm{g} / \mathrm{mL}$.

\section{Statistical Analysis}

All the experiments were performed in triplicate. Data of experimental results was recorded as mean \pm standard deviation. One way ANOVA $t$-test was used for statistical significance and a $P$-value $<0.05$ (denoted as ${ }^{*}$ ) was regarded as significant. Correlation coefficient $(r)$ value calculated by using Pearson's
Correlation method. Statistical analyses were performed using Graph Pad Prism 5.0 statistical software.

\section{RESULTS}

\section{MICs of Tulathromycin and Gallic Acid}

The MIC of tulathromycin was determined to be $0.31 \mu \mathrm{g} / \mathrm{mL}$ against both $M$. haemolytica and $P$. multocida (Figures 1A,B). The MIC of gallic acid was determined to be $250 \mu \mathrm{g} / \mathrm{mL}$ against $M$. haemolytica (Figure 1C) and $500 \mu \mathrm{g} / \mathrm{mL}$ against $P$. multocida (Figure 1D).

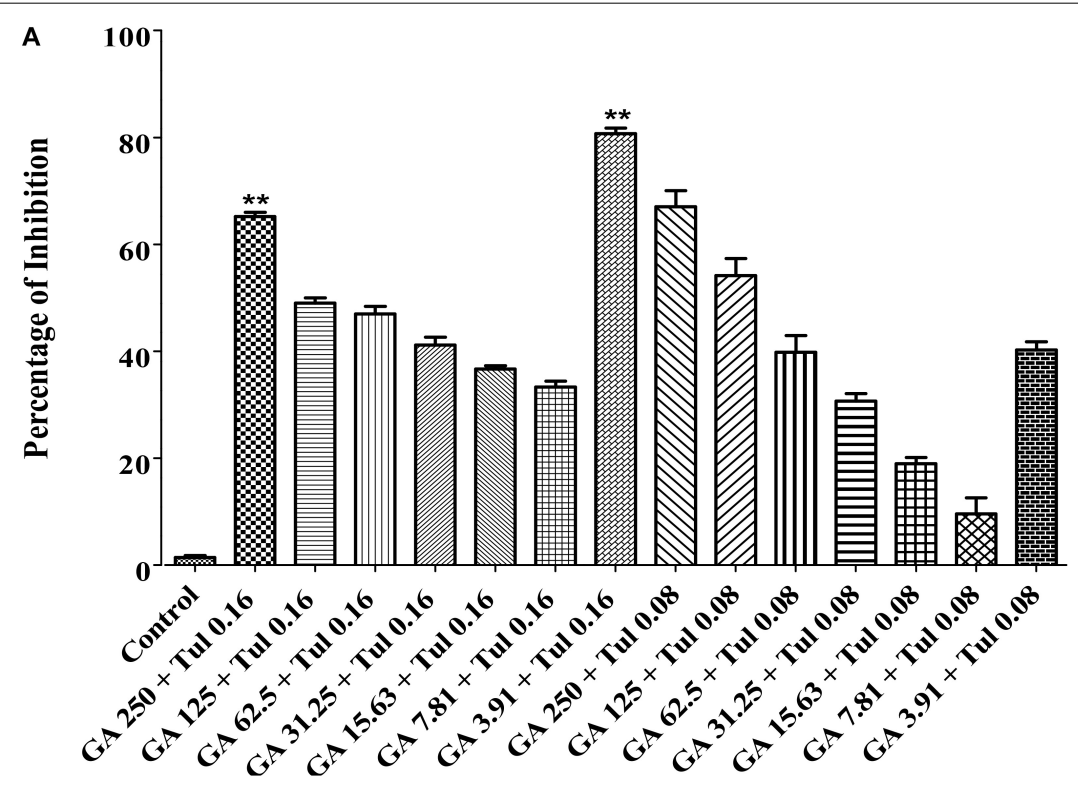

B

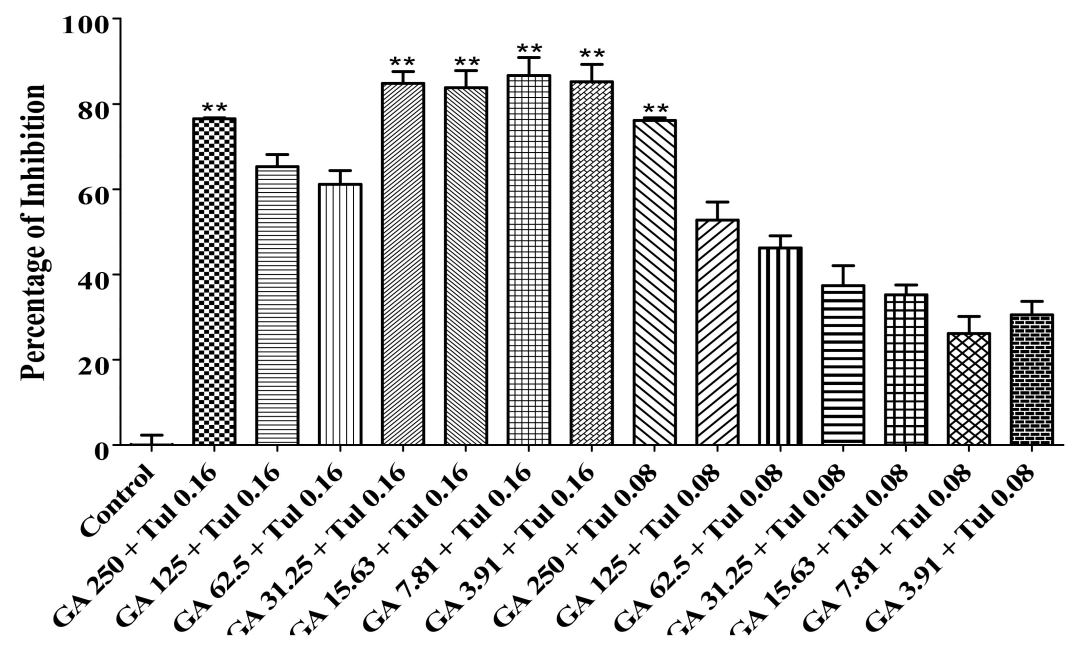

FIGURE 2 | Antimicrobial activity of the combination of tulathromycin and gallic acid against $M$. haemolytica (A) and $P$. multocida (B). The concentration of tulathromycin was 0.08 and $0.16 \mu \mathrm{g} / \mathrm{mL}$, respectively, and the concentration of gallic acid was from 3.91 to $250 \mu \mathrm{g} / \mathrm{mL}$. Bacterial cell culture medium, BHIB, was used as a negative control. One way ANOVA $t$-test was used for statistical significance. ${ }^{*} P$-value $<0.05,{ }^{* *} P$-value $<0.01$, and ${ }^{* * *} P$-value $<0.001$ are shown. Correlation coefficient $(r)$ was calculated using Pearson's correlation method. 


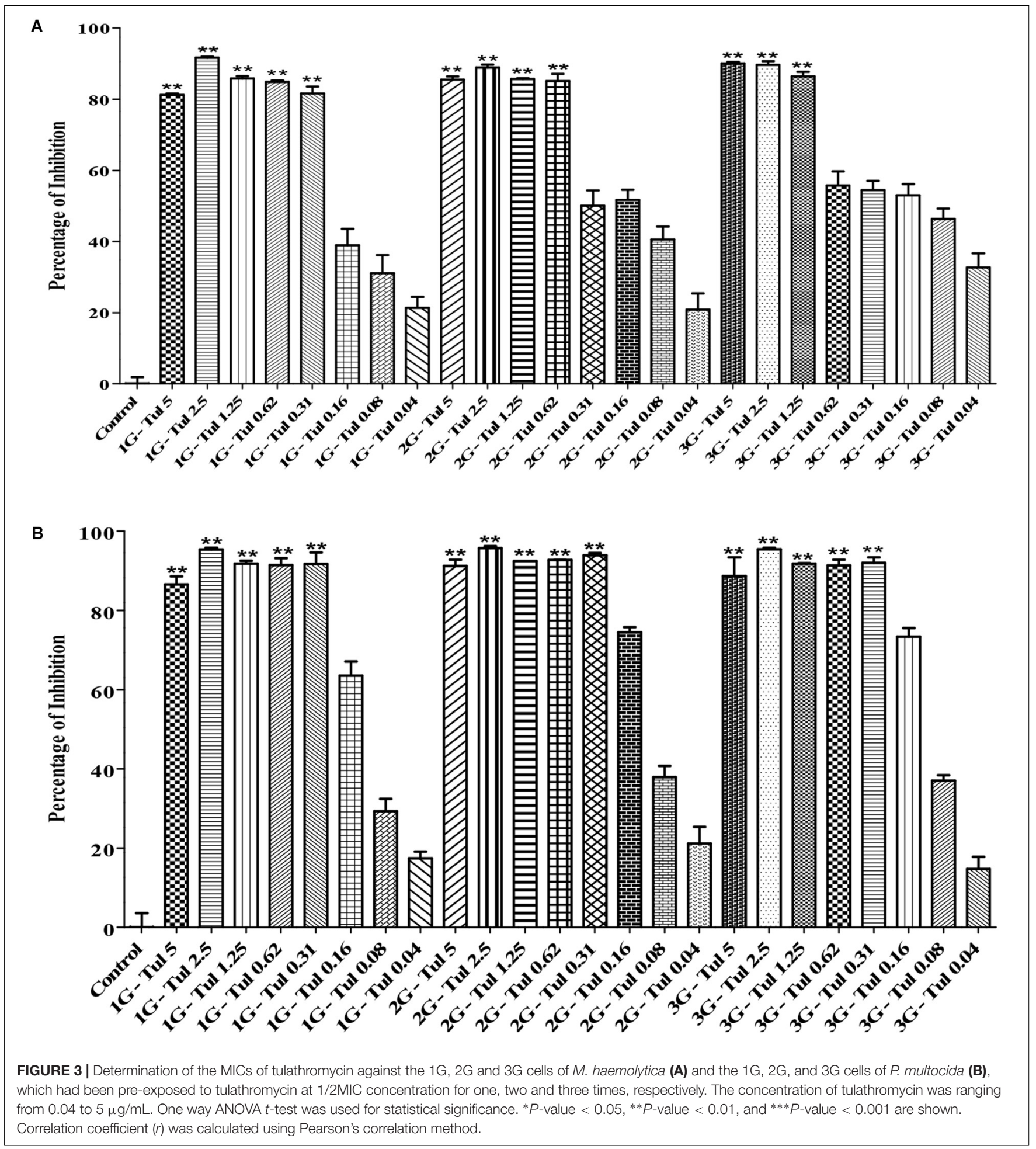

\section{Strong Additive or Weak Synergistic Effect Between Tulathromycin and Gallic Acid}

The checkerboard broth micro-dilution assay was carried out to investigate whether co-administration of tulathromycin and gallic acid could exhibit synergistic/additive effect. The antimicrobial activity against $M$. haemolytica and $P$. multocida was evaluated for tulathromycin at two sub-MIC doses $(0.16$ and $0.08 \mu \mathrm{g} / \mathrm{mL})$, gallic acid at seven sub-MIC doses $(250,125,62.5$, $31.25,15.6,7.8$, and $3.9 \mu \mathrm{g} / \mathrm{mL}$ ), and their combination. For M. haemolytica, tulathromycin gave a $37 \%$ inhibition of growth 
at concentration of $0.16 \mu \mathrm{g} / \mathrm{mL}$ and gallic acid showed an $8 \%$ inhibition of growth at concentration of $3.91 \mathrm{~g} / \mathrm{mL}$ (Figure 2A). However, co-administration of $0.16 \mu \mathrm{g} / \mathrm{mL}$ tulathromycin and $3.91 \mu \mathrm{g} / \mathrm{mL}$ gallic acid dramatically increased the inhibition of growth to $81 \%$ (Figure 2A). The FIC index of the combination of tulathromycin and gallic acid was 0.5 , implicating a strong additive or weak synergistic effect between tulathromycin and gallic acid agsint $M$. haemolytica. For P. multocida, tulathromycin exhibited a $77 \%$ inhibition of growth at concentration of $0.16 \mu \mathrm{g} / \mathrm{mL}$ and gallic acid gave a $5 \%$ inhibition of growth at concentration of $3.91 \mu \mathrm{g} / \mathrm{mL}$ (Figure $2 B$ ). Co-administration of $0.16 \mu \mathrm{g} / \mathrm{mL}$ tulathromycin and $3.91 \mu \mathrm{g} / \mathrm{mL}$ gallic acid enhanced the inhibition of growth to $91 \%$ (Figure 2B). The FIC index of the combination of tulathromycin and gallic acid was calculated to be 0.5 , implicating that there is also a strong additive or weak synergistic effect between tulathromycin and gallic acid agsint P. multocida. The mixed culture of $M$. haemolytica and P. multocida, tulathromycin gave a $70.6 \%$ inhibition of growth at concentration of $0.16 \mu \mathrm{g} / \mathrm{mL}$ (Figure 5A). However, gallic acid showed $38.5 \%$ inhibition of growth at a concentration of $31.25 \mu \mathrm{g} / \mathrm{mL}$ (Figure 5B). Co-administration of $0.16 \mu \mathrm{g} / \mathrm{mL}$ tulathromycin and $31.25 \mu \mathrm{g} / \mathrm{mL}$ gallic acid reasonably increased the inhibition of growth to $74.2 \%$ (Figure $5 \mathrm{C}$ ). The FIC index of the combination of tulathromycin and gallic acid was 0.56 suggesting a strong additive or weak synergistic effect between tulathromycin and gallic acid against the mixed culture of $M$. haemolytica and P. multocida. Other combinations showed indifferent effect.

\section{Bacterial Resistance Generated From Pre-exposure}

In this study, we generated three generations (1G, 2G, and 3G) of M. haemolytica and P. multocida samples that had been exposed to either tulathromycin or gallic acid for one, two and three times, respectively. As shown in Figure 3, the MIC of tulathromycin was measured to be $0.31 \mu \mathrm{g} / \mathrm{mL}$ for $1 \mathrm{G}, 0.62 \mu \mathrm{g} / \mathrm{mL}$ for $2 \mathrm{G}$, and $1.25 \mu \mathrm{g} / \mathrm{mL}$ for $3 \mathrm{G}$, respectively, for $M$. haemolytica and $0.31 \mathrm{~g} / \mathrm{mL}$ for all three generations of pre-exposed $P$. multocida. This implicated that pre-exposure to tulathromycin at $1 / 2 \mathrm{MIC}(0.16 \mu \mathrm{g} / \mathrm{mL})$ induced bacterial resistance in $M$. haemolytica but not in $P$. multocida. Furthermore, the bacterial resistance was positively correlated with the pre-exposure times of tulathromycin for M. haemolytica. For gallic acid, the MIC was measured to be $250 \mu \mathrm{g} / \mathrm{mL}$ for all three generations of pre-exposed $M$. haemolytica and

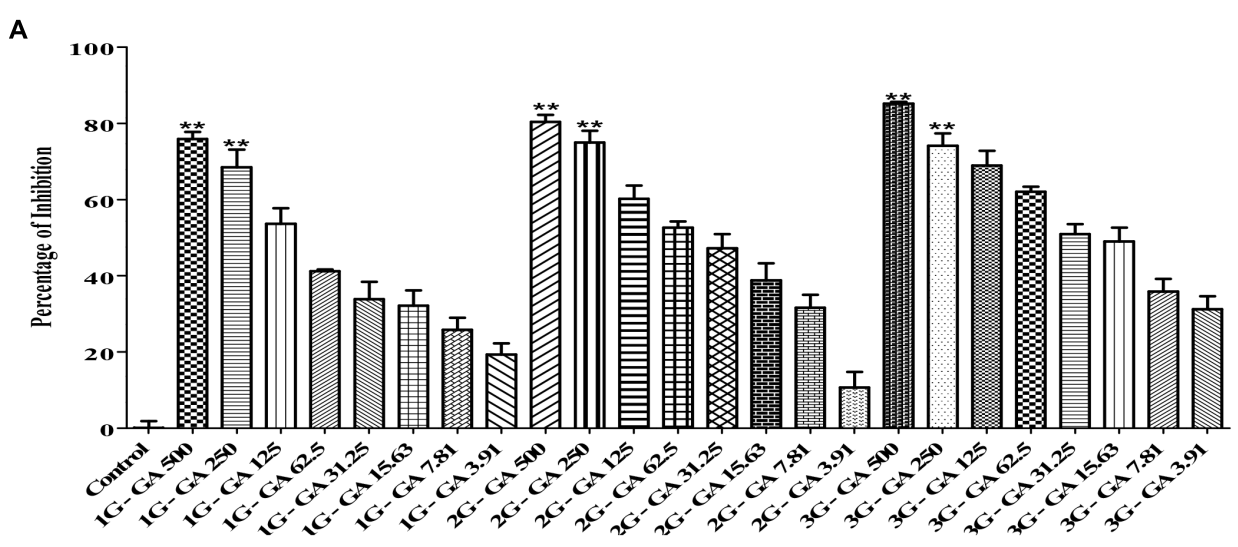

B

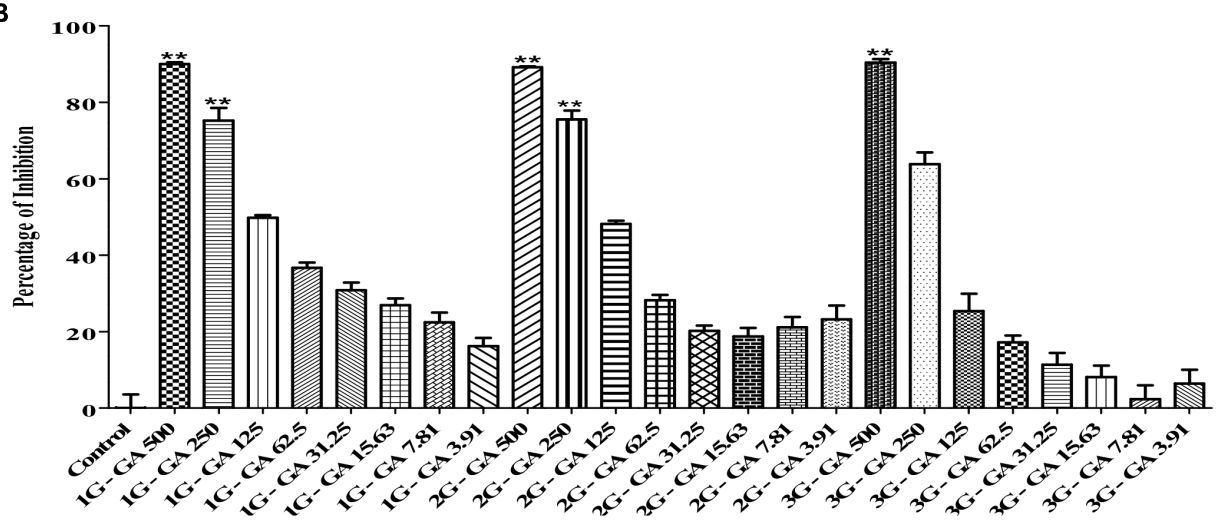

FIGURE 4 | Determination of the MICs of gallic acid against the 1G,2G, and $3 \mathrm{G}$ cell of $M$. haemolytica (A) and the 1G, $2 \mathrm{G}$ and $3 \mathrm{G}$ cells of $P$. multocida (B), which had been pre-exposed to tulathromycin at 1/2MIC concentration for one, two, and three times, respectively. The concentration of gallic acid was ranging from 3.9 to $500 \mu \mathrm{g} / \mathrm{mL}$. One way ANOVA $t$-test was used for statistical significance. ${ }^{*} P$-value $<0.05,{ }^{* *} P$-value $<0.01$, and ${ }^{* * *} P$-value $<0.001$ are shown. Correlation coefficient $(r)$ was calculated using Pearson's correlation method. 

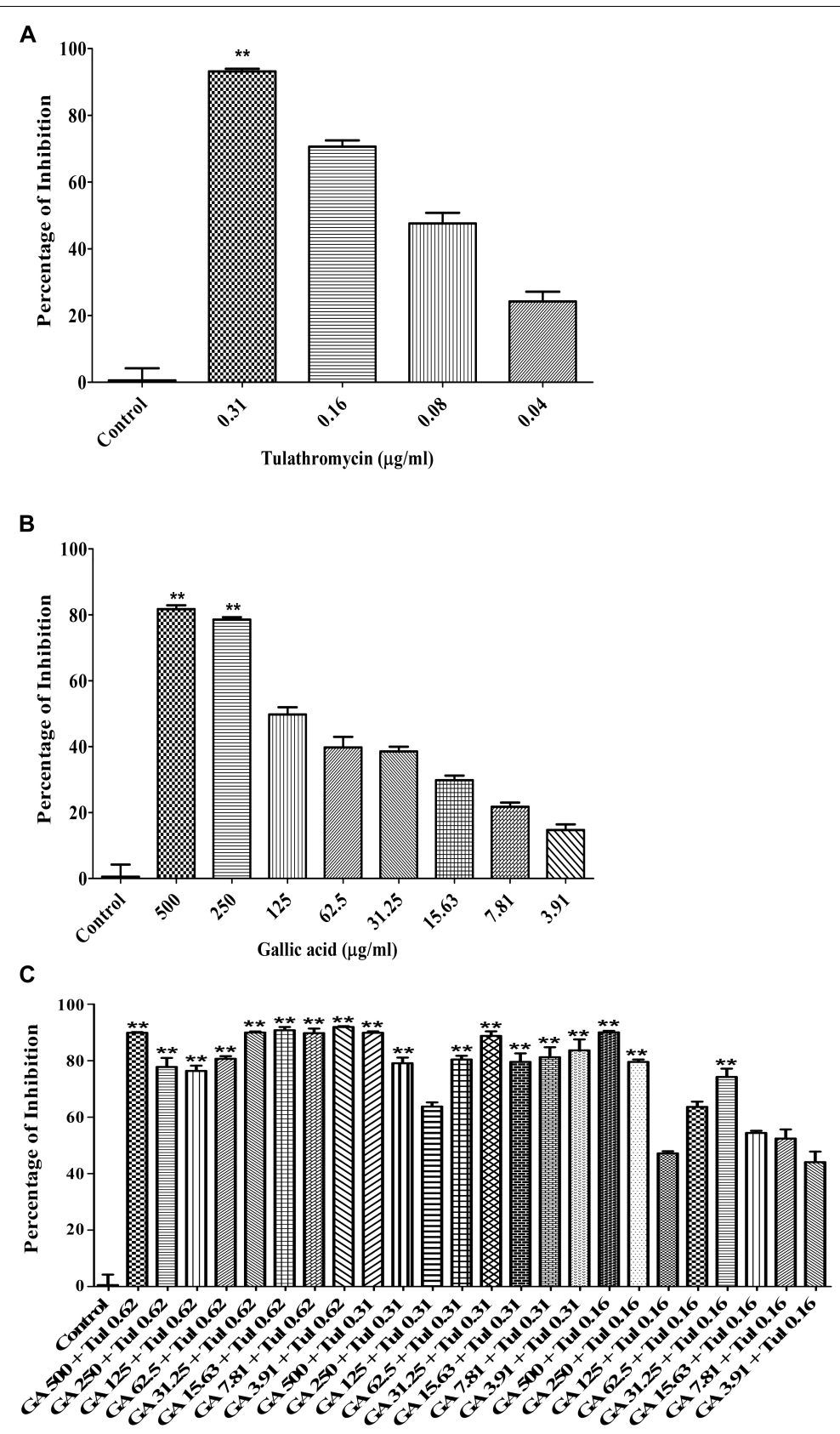

FIGURE 5 | Antimicrobial activity of tylathromycin [(A) concentration: $0.04-0.31 \mu \mathrm{g} / \mathrm{mL}$ ], gallic acid [(B) concentration: $3.91-500 \mu \mathrm{g} / \mathrm{mL}]$ and combination of tulathromycin and gallic acid [(C) concentration: tulathromycin $0.16,0.31$, and $0.62 \mu \mathrm{g} / \mathrm{mL}$ and gallic acid $3.91-500 \mu \mathrm{g} / \mathrm{mL}]$ against the mixed culture of $M$. haemolytica and $P$. multocida. One way ANOVA $t$-test was used for statistical significance. ${ }^{*} P$-value $<0.05,{ }^{* *} P$-value $<0.01$, and ${ }^{* * *} P$-value $<0.001$ are shown. Correlation coefficient ( $r$ ) was calculated using Pearson's correlation method.

$250 \mu \mathrm{g} / \mathrm{mL}$ for $1 \mathrm{G}, 250 \mu \mathrm{g} / \mathrm{mL}$ for $2 \mathrm{G}$, and $500 \mu \mathrm{g} / \mathrm{mL}$ for $3 G$, respectively, for $P$. multocida (Figure 4). Therefore, pre-exposure to tulathromycin did not alter the sensitivity of $M$. haemolytica but sensitized $P$. multocida toward gallic acid. Further studies are warranted to confirm whether this sensitization toward gallic acid is true and how it has happened in P. multocida.

Absorbance data is available upon request.

\section{DISCUSSION}

Bovine respiratory disease (BRD), which is associated with morbidity and mortality, is the most common infectious disease in cattle and a significant threat to dairy and beef industry. Mass antimicrobial treatment upon animal arrival is widely used to reduce incidence of $\mathrm{BRD}$ and eliminate/minimize a potential outbreak (Taylor et al., 2010). However, the increased global 
concern on antimicrobial abuse in the dairy and beef industry has led many countries to ban or limit antimicrobials as growth promoters (McVey, 2009; Urban-Chmiel and Grooms, 2012; Johnston et al., 2017). In western Canada, tulathromycin is normally used as the metaphylactic agent to reduce the risk and severity of BRD. Resistance to tulathromycin, conferred by rRNA mutations, has been found in field isolates of M. haemolytica and P. multocida (Wadood et al., 2017; Uddin et al., 2018). Therefore, it is important to develop a novel strategy to decrease the dosage of tulathromycin, which, in turn, would reduce or eliminate the generation of resistance by the bacterial pathogens. One effective way to reduce antimicrobial dosage and delay AMR is to use a cocktail of two antimicrobials or a cocktail of an antimicrobial and a phytochemical.

Phytochemicals, which are antimetabolites produced in plants, have provided a valuable resource for relatively cheap and safe antimicrobial agents. The toxicity of phytochemicals is normally low and very few adverse effects have been reported (Nascimento et al., 2000). It has been shown that gallic acid, a phenolic acid, not only possesses antimicrobial activity against various bacteria such as Pseudomonas strains but also potentiates the efficacy of antimicrobials (Al-Abd et al., 2015; Fu et al., 2016; Samad et al., 2016). Previous studies have also demonstrated that gallic acid inhibited efflux pumps, which are a major mechanism in generating AMR, in Staphylococcus aureus resistant strains and multidrug resistant Escherichia coli strains (Simoes et al., 2009; Abreu et al., 2012). In addition, epigallocatechin gallate, which is an ester of epigallocatechin and gallic acid, has been shown to exhibit antifolate activity against Stenotrophomonas maltophilia (Navarro-Martínez et al., 2005). Therefore, the strong additive or weak synergistic effect between tulathromycin and gallic acid against $M$. haemolytica and P. multocida is likely through the inhibitory effect of gallic acid on efflux pumps and antifolate activity. Further studies are warranted to confirm whether gallic acid can indeed inhibit efflux pumps and folate synthesis in M. haemolytica and P. multocida. It is also noteworthy that gallic acid is an arginase inhibitor and arginase has been shown to be regulated in allergic lung disease (Pudlo et al., 2017). Since controlling pulmonary inflammation with an NSAID is critical to avoid irreversible lung damage in the cattle infected with BRD (Pal et al., 2010), the anti-inflammatory activity of gallic acid via inhibiting arginase would provide an extra benefit in treating $\mathrm{BRD}$.

Because BRD is a multi-factorial syndrome and more than one bacteria may be involved in the disease simultaneously,

\section{REFERENCES}

Abreu, A. C., Mcbain, A. J., and Simoes, M. (2012). Plants as sources of new antimicrobials and resistance-modifying agents. Nat. Prod. Rep. 29, 1007-1021. doi: $10.1039 / \mathrm{c} 2 \mathrm{np} 20035 \mathrm{j}$

Al-Abd, N. M., Nor, Z. M., Mansor, M., Azhar, F., Hasan, M. S., and Kassim, M. (2015). Antioxidant, antibacterial activity, and phytochemical characterization of Melaleuca cajuputi extract. BMC Complement. Altern. Med. 15:385. doi: 10.1186/s12906-015-0914-y

Amyes, S. G., Walsh, F. M., and Bradley, J. S. (2007). Best in class: a good principle for antibiotic usage to limit resistance development? J. Antimicrob. Chemother. 59, 825-826. doi: 10.1093/jac/dkm059 we decided to evaluate whether tulathromycin, gallic acid, and/or their combination were effective against the mixed culture of M. haemolytica and P. multocida. The MIC of tulathromycin was found to be $0.31 \mu \mathrm{g} / \mathrm{mL}$ against the mixed culture (Figure 5A), which is the same as that against each individual bacterium. The MIC of gallic acid was found to be $500 \mu \mathrm{g} / \mathrm{mL}$ against the mixed culture (Figure 5B), which is the same as that against $P$. multocida but higher than that against M. haemolytica. Co-administration of $0.16 \mu \mathrm{g} / \mathrm{mL}$ tulathromycin and $500 \mu \mathrm{g} / \mathrm{mL}$ gallic acid produced the maximum inhibition of the mixed culture (Figure 5C). Therefore, we concluded that tulathromycin, gallic acid and their combination are also effective against the mixed culture of $M$. haemolytica and P. multocida. Further validation of the current data with clinical isolates of $M$. haemolytica and P. multocida and investigation with an in vivo animal model may help in determining whether the co-administration approach with gallic acid is indeed clinically practical in reducing dosage of tulathromycin and delay/prevent the emergence of AMR in feedlots.

\section{CONCLUSION}

Bovine respiratory disease (BRD) is the most common infectious disease in dairy and beef cattle. In this study, we showed that both tulathromycin and gallic acid were effective against M. haemolytica, P. multocida, and their mixed culture. A strong additive or weak synergistic effect was observed between tulathromycin and gallic acid against both bacteria. In addition, pre-exposure to tulathromycin generated bacterial resistance to the antimicrobial in M. haemolytica but not in P. multocida.

\section{AUTHOR CONTRIBUTIONS}

This work was designed by MS and JY carried out by KR. The manuscript was prepared by MS and JY and approved by all authors.

\section{FUNDING}

This work was supported in part by an Agricultural Development Fund (ADF) grant from the Province of Saskatchewan, Canada (418897), and four Accelerate Internships from Mitacs Canada, and SaskMilk (Regina, SK, Canada) (419249).

Andersson, I. D., and Hughes, D. (2014). Microbiological effects of sublethal levels of antibiotics. Nat. Rev. Microbiol. 12, 465-478. doi: 10.1038/nrmicro 3270

Apley, M. (2006). Bovine respiratory disease: pathogenesis, clinical signs, and treatment in lightweight calves. Vet. Clin. North Am. Food Anim. Pract. 22, 399-411. doi: 10.1016/j.cvfa.2006.03.009

Batista, O., Duarte, A., Nascimento, J., Simões, M. F., De La Torre, M. C., and Rodríguez, B. (1994). Structure and antimicrobial activity of diterpenes from the roots of Plectranthus hereroensis. J. Nat. Prod. 57, 858-861. doi: 10.1021/ np50108a031

Bhattacharya, G., Dey, D., Das, S., and Banerjee, A. (2017). Exposure to subinhibitory concentrations of gentamycin, ciprofloxacin and cefotaxime induces 
multidrug resistance and reactive oxygen species generations in meticillinsensitive Staphylococcus aureus. J. Med. Microbiol. 66, 762-769. doi: 10.1099/ jmm.0.000492

Cai, Y., Wang, R., Pei, F., and Liang, B.-B. (2007). Antibacterial activity of allicin alone and in combination with $\beta$-lactams against Staphylococcus spp. and Pseudomonas aeruginosa. J. Antibiot. 60, 335-338. doi: 10.1038/ja.2007.45

Černohorská, L., and Votava, M. (2008). Antibiotic synergy against biofilmforming Pseudomonas aeruginosa. Folia Microbiol. 53, 57-60. doi: 10.1007/ s12223-008-0008-z

Checkley, S. L., Campbell, J. R., Chirino-Trejo, M., Janzen, E. D., and Waldner, C. L. (2010). Associations between antimicrobial use and the prevalence of antimicrobial resistance in fecal Escherichia coli from feedlot cattle in western Canada. Can. Vet. J. 51, 853-861.

Cowan, M. M. (1999). Plant products as antimicrobial agents. Clin. Microbiol. Rev. 12, 564-582. doi: 10.1128/CMR.12.4.564

Ellis, J. A. (2001). The immunology of the bovine respiratory disease complex. Vet. Clin. North Am. Food Anim. Pract. 17, 535-550. doi: 10.1016/S0749-0720(15) 30005-0

Fu, L., Lu, W., and Zhou, X. (2016). Phenolic compounds and in vitro antibacterial and antioxidant activities of three tropic fruits: persimmon, guava, and sweetsop. BioMed. Res. Int. 2016, 1-9. doi: 10.1155/2016/4287461

Gould, I. (2008). Antibiotic policies to control hospital-acquired infection. J. Antimicrob. Chemother. 61, 763-765. doi: 10.1093/jac/dkn039

Griffin, D. (1997). Economic impact associated with respiratory disease in beef cattle. Vet. Clin. North Am. Food Anim. Pract. 13, 367-377. doi: 10.1016/S07490720(15)30302-9

Headley, S. A., Okano, W., Balbo, L. C., Marcasso, R. A., Oliveira, T. E., Alfieri, A. F., et al. (2018). Molecular survey of infectious agents associated with bovine respiratory disease in a beef cattle feedlot in southern Brazil. J. Vet. Diagn. Invest. 30, 249-251. doi: 10.1177/1040638717739945

Holman, D. B., Klima, C. L., Ralston, B. J., Niu, Y. D., Stanford, K., Alexander, T. W., et al. (2017). Metagenomic sequencing of bronchoalveolar lavage samples from feedlot cattle mortalities associated with bovine respiratory disease. Genome Announc. 5:e01045-17. doi: 10.1128/genomeA.01045-17

Johnson, K. K., and Pendell, D. L. (2017). Market impacts of reducing the prevalence of bovine respiratory disease in United States beef cattle feedlots. Front. Vet. Sci. 4:189. doi: 10.3389/fvets.2017.00189

Johnston, D., Earley, B., Cormican, P., Murray, G., Kenny, D. A., Waters, S. M., et al. (2017). Illumina MiSeq 16S amplicon sequence analysis of bovine respiratory disease associated bacteria in lung and mediastinal lymph node tissue. BMC Vet. Res. 13:118. doi: 10.1186/s12917-017-1035-2

Larson, R. (2005). Effect of cattle disease on carcass traits 1. J. Anim. Sci. 83, E37-E43.

Larson, R. L., and Step, D. (2012). Evidence-based effectiveness of vaccination against Mannheimia haemolytica, Pasteurella multocida, and Histophilus somni in feedlot cattle for mitigating the incidence and effect of bovine respiratory disease complex. Vet. Clin. North Am. Food Anim. Pract. 28, 97-106. doi: 10.1016/j.cvfa.2011.12.005

Lewis, K., and Ausubel, F. M. (2006). Prospects for plant-derived antibacterials. Nat. Biotechnol. 24, 1504-1507. doi: 10.1038/nbt1206-1504

Lindsay, M. A. (2003). Target discovery. Nat. Rev. Drug Discov. 2, 831-838. doi: $10.1038 / \mathrm{nrd} 1202$

McVey, D. S. (2009). BRD research needs in the next 10-20 years. Anim. Health Res. Rev. 10, 165-167. doi: 10.1017/S1466252309990247

Monden, K., Ando, E., Iida, M., and Kumon, H. (2002). Role of fosfomycin in a synergistic combination with ofloxacin against Pseudomonas aeruginosa growing in a biofilm. J. Infect. Chemother. 8, 218-226. doi: 10.1007/s10156-0020186-6

Nascimento, G. G., Locatelli, J., Freitas, P. C., and Silva, G. L. (2000). Antibacterial activity of plant extracts and phytochemicals on antibiotic-resistant bacteria. Braz. J. Microbiol. 31, 247-256. doi: 10.1590/S1517-83822000000400003

Navarro-Martínez, M. D., Navarro-Perán, E., Cabezas-Herrera, J., Ruiz-Gómez, J., García-Cánovas, F., and Rodríguez-López, J. N. (2005). Antifolate activity of epigallocatechin gallate against Stenotrophomonas maltophilia. Antimicrob. Agents Chemother. 49, 2914-2920. doi: 10.1128/AAC.49.7.2914-2920.2005
Neu, H. (1991). Synergy and antagonism of combinations with quinolones. Eur. J. Clin. Microbiol. Infect. 10, 255-261. doi: 10.1007/BF01966998

Pal, C., Bindu, S., Dey, S., Alam, A., Goyal, M., Iqbal, M. S., et al. (2010). Gallic acid prevents nonsteroidal anti-inflammatory drug-induced gastropathy in rat by blocking oxidative stress and apoptosis. Free Radic. Biol. Med. 49, 258-267. doi: 10.1016/j.freeradbiomed.2010.04.013

Petersen, P. J., Labthavikul, P., Jones, C. H., and Bradford, P. A. (2006). In vitro antibacterial activities of tigecycline in combination with other antimicrobial agents determined by chequerboard and time-kill kinetic analysis. J. Antimicrob. Chemother. 57, 573-576. doi: 10.1093/jac/dki477

Pudlo, M., Demougeot, C., and Girard-Thernier, C. (2017). Arginase inhibitors: a rational approach over one century. Med. Res. Rev. 37, 475-513. doi: 10.1002/ med.21419

Rajala-Schultz, P., Torres, A., Degraves, F., Gebreyes, W., and Patchanee, P. (2009). Antimicrobial resistance and genotypic characterization of coagulase-negative staphylococci over the dry period. Vet. Microbiol. 134, 55-64. doi: 10.1016/j. vetmic.2008.09.008

Samad, M. A., Hashim, S. H., Simarani, K., and Yaacob, J. S. (2016). Antibacterial properties and effects of fruit chilling and extract storage on antioxidant activity, total phenolic and anthocyanin content of four date palm (Phoenix dactylifera) cultivars. Molecules 21:419. doi: 10.3390/molecules21040419

Schunicht, O. C., Booker, C. W., Guichon, P. T., Jim, G. K., Wildman, B. K., Pittman, T. J., et al. (2007). An evaluation of the relative efficacy of tulathromycin for the treatment of undifferentiated fever in feedlot calves in Nebraska. Can. Vet. J. 48, 600-606.

Simoes, M., Bennett, R. N., and Rosa, E. A. (2009). Understanding antimicrobial activities of phytochemicals against multidrug resistant bacteria and biofilms. Nat. Prod. Rep. 26, 746-757. doi: 10.1039/b821648g

Smith, R. A. (1998). Impact of disease on feedlot performance: a review. J. Anim. Sci. 76, 272-274. doi: 10.2527/1998.761272x

Taylor, J. D., Fulton, R. W., Lehenbauer, T. W., Step, D. L., and Confer, A. W. (2010). The epidemiology of bovine respiratory disease: what is the evidence for predisposing factors? Can. Vet. J. 51, 1095-1102.

Uddin, R., Siddiqui, Q. N., Azam, S. S., Saima, B., and Wadood, A. (2018). Identification and characterization of potential druggable targets among hypothetical proteins of extensively drug resistant Mycobacterium tuberculosis (XDR KZN 605) through subtractive genomics approach. Eur. J. Pharm. Sci. 114, 13-23. doi: 10.1016/j.ejps.2017.11.014

Urban-Chmiel, R., and Grooms, D. (2012). Prevention and control of bovine respiratory disease. J. Livest. Sci. 3, 27-36.

Wadood, A., Jamal, A., Riaz, M., Khan, A., Uddin, R., Jelani, M., et al. (2017). Subtractive genome analysis for in silico identification and characterization of novel drug targets in Streptococcus pneumonia strain JJA. Microb. Pathog. 115, 194-198. doi: 10.1016/j.micpath.2017.12.063

West-Central Forage Association (2018). This December, You Will Need a Prescription to Buy Virtually any Livestock Antibiotic. 2018. Entwistle, AB: West-Central Forage Association.

Wise, R. (2006). Antimicrobial resistance: paradox, actions and economics. J. Antimicrob. Chemother. 57, 1024-1025. doi: 10.1093/jac/dkl138

Wittum, T., Woollen, N., Perino, L., and Littledike, E. (1996). Relationships among treatment for respiratory tract disease, pulmonary lesions evident at slaughter, and rate of weight gain in feedlot cattle. J. Am. Vet. Med. Assoc. 209, 814-818.

Conflict of Interest Statement: The authors declare that the research was conducted in the absence of any commercial or financial relationships that could be construed as a potential conflict of interest.

Copyright (C) 2019 Rajamanickam, Yang and Sakharkar. This is an open-access article distributed under the terms of the Creative Commons Attribution License (CC BY). The use, distribution or reproduction in other forums is permitted, provided the original author(s) and the copyright owner(s) are credited and that the original publication in this journal is cited, in accordance with accepted academic practice. No use, distribution or reproduction is permitted which does not comply with these terms. 